\title{
COMMENTARY
}

\section{The Growing Field of Nanomedicine and Its Relevance to Pharmacy Curricula}

\author{
Volkmar Weissig, ${ }^{\mathrm{a}}$ Tamer Elbayoumi, ${ }^{\mathrm{a}}$ Beat Flühmann, ${ }^{\mathrm{b}}$ Amy Barton ${ }^{\mathrm{b}}$ \\ ${ }^{a}$ Midwestern University, College of Pharmacy Glendale, Glendale, Arizona \\ ${ }^{\mathrm{b}}$ Vifor Pharma Group, Vifor Pharma Management Ltd, Glattbrugg, Switzerland
}

Corresponding Author: Volkmar Weissig, Midwestern University, College of Pharmacy Glendale, 19555 N. $59^{\text {th }}$ Avenue, Glendale, AZ 85308. Email: vweiss@ midwestern.edu

Submitted August 5, 2020; accepted February 10, 2021; ePublished February 2021

The field of nanomedicine is a rapidly growing scientific domain. Nanomedicine encompasses a diverse number of active pharmaceutical ingredients. Submissions of Investigational New Drugs and New Drug Applications have risen dramatically over the last decade. Currently, there are over 50 FDA approved nanomedicines on the US market. Thus, because of the fundamental role pharmacists will play in therapeutic and administrative decisions regarding nanomedicines, it is imperative that they gain exposure to this rapidly evolving class of drugs. This commentary will describe nanomedicines, discuss current regulatory challenges, and provide recommendations for judicious incorporation of nanomedicine topics into the Doctor of Pharmacy curriculum based on emerging pharmaceutical and clinical science applications.

Keywords: nanomedicine; pharmaceutics; student pharmacist

\section{INTRODUCTION}

Significant advances in nanoscale material science during the last quarter of the last century have spurred the development of nanotechnology as an independent scientific discipline and its merger with medicine and pharmacy around the turn of the century brought about the advent of Nanomedicines. Currently, there are over 50 nanomedicines (referred to by the Food and Drug Administration (FDA) as drugs containing nanomaterials) on the US market. ${ }^{1}$ In comparison to their small molecule parent drugs, nanomedicines display different and complex pharmacokinetic (PK) and pharmacodynamic profiles and subsequently possess different safety and therapeutic efficacy. In keeping with their broad recommendations for pharmacy curricula, the Center for the Advancement for Pharmaceutical Education (CAPE) Outcomes suggest covering "evaluation of future advances in medicine". Thus, judicious incorporation of nanomedicine topics into pharmacy curricula appears warranted.

\section{History and Definition of Nanomedicines (Nanopharmaceuticals)}

The term "Nanotechnology" was coined and used for the very first time in 1974 by Norio Taniguchi. ${ }^{2}$ Based on many more major discoveries and developments in nanoscale material science, the National Institutes of Health (NIH) launched the National Nanotechnology Initiative in the year 2000., ${ }^{3}$ This federal program facilitated nanoscale-related interdisciplinary research which quickly led to the well-funded merger between nanotechnology and medicine, giving rise to nanomedicine as a new scientific discipline. The NIH defines Nanotechnology as "The understanding and control of matter at dimensions between approximately 1 and 100 nanometers $(\mathrm{nm})$, where unique phenomena enable novel applications". ${ }^{3}$ Drug products containing nanomaterials may be developed as active ingredients or carriers loaded with active or inactive ingredients. ${ }^{4,5}$ Emphasizing the unique nanoscale properties and expanding the size scale beyond 100 $\mathrm{nm}$, the FDA also suggests that a drug product can be considered a nanomaterial if the material or end product is engineered to exhibit specific properties or phenomena and that these features are attributable to its dimension(s), even if these dimensions fall outside the nanoscale range, up to one micrometer $(1,000 \mathrm{~nm}) .^{5} \mathrm{In}$ addition to the nanomedicines currently in clinical use, there are many nanomedicines that are presently in different phases of pre-clinical and clinical development. ${ }^{6,7}$ This includes products exhibiting a potential therapeutic effect based on their unique nanoscale physicochemical properties such as superparamagnetism exhibited by iron oxide nanoparticles or improved bioavailability and disease specificity with lipid drug delivery carriers such as those that have been utilized for FDA approved COVID19 vaccines. ${ }^{8}$ The growth of the field of nanomedicines is also exemplified by a rapid growth of indexed publications (Figure 1). Correspondingly, according to an analysis by the Center for Drug Evaluation and Research (CDER, USA), since 1970 there have been more than 600 of investigational new drug (IND), new drug (NDA) and abbreviated new drug 
(ANDA) applications for human drug products containing nanomaterials with half of these applications being submitted during the last decade. ${ }^{4}$ The widespread clinical use of nanomedicines is also demonstrated by sales of liposomal doxorubicin which is projected to be 1.39 billion by $2024 .{ }^{9}$ A recent analysis of Medicare Part B expenditures from 20062017 showed the total spending for the nanomedicine Abraxane (protein-bound paclitaxel) was more than 100 times that of the standard small molecule formulation of paclitaxel. ${ }^{10}$

\section{Types of Nanomedicines}

The majority of nanomedicines in clinical use are based on liposomes. Liposomal encapsulated doxorubicin ("Doxil") is considered the very first FDA-approved nanomedicine and was approved in 1995. ${ }^{11}$ Liposomal doxorubicin has been shown to be good alternative to paclitaxel in combination with carboplatin as a first line treatment for epithelial ovarian cancer and is associated with reduced risk of alopecia and neurotoxicity. ${ }^{12}$ Other liposomal nanomedicines include AmBisome, Depocyt, Myocet and DepoDur. Liposomal Amphotericin B is recommended as a first-line therapy for invasive, restricted compartment infections such as mucormycosis due to its enhanced ability to penetrate target tissues while delaying relevant toxicities (eg, acute kidney injury). ${ }^{13}$ Proteins, polypeptides and aptamers which have been covalently linked to a varying number of polyethylene glycol (PEG) chains display very different PK and possess a totally altered bioavailability in comparison to their non-PEGylated parent molecules. Examples are Adagen, Eulasta, Oncaspar and Somavert. Nanocrystals constitute a unique group of nanomedicines since they are entirely composed of waterinsoluble drug substance without any other added nanomaterials. Emend, Rapamune and Tricor are examples. Polymerbased nanoformulations involve Copaxone, Eligard, Genexol and Opaxio. Protein-drug conjugates are using common proteins like albumin as nanodrug carriers as in Abraxane, which is paclitaxel linked to albumin nanoparticles. Another group of nanomedicines is based on metal nanoparticles. The first iron carbohydrate nanomedicine, Venofer was approved in Switzerland since 1949, and later, Ferrlecit, Injectafer and Feraheme All of these colloidal solutions are comprised of iron carbohydrate nanoparticles, and are approved for intravenous treatment of iron deficiency and iron deficiency anemia. A schematic overview of the major types of nanomedicines is shown in Figure 2 and for a comprehensive discussion of their composition we refer the reader to previously published reviews. ${ }^{7,8}$

Nanomedicines belong to a class defined by the FDA as Complex Drugs which acknowledges that evaluation of nanomedicines poses unique challenges compared to small molecule medicines. ${ }^{5}$ Characterizing nanomedicines is inherently challenging due to their structural complexity and heterogeneity, which limits the utility of traditional analytic chemistry techniques in fully describing the physicochemical characteristics of nanomedicines. ${ }^{14,15}$ For example, some experimental conditions (eg, dilution) can alter or degrade the intact nanoparticle structure. ${ }^{14}$ Additionally, there are no standards for many new physicochemical characterization techniques, however, these efforts are ongoing. ${ }^{14,15}$ Because nanomedicines are designed to target specific tissues and cell types, simple plasma PK profiles may not accurately predict the biodistribution profiles. ${ }^{16}$ Additionally, there are few assays that can distinguish the free from encapsulated active drug. ${ }^{17}$ All of these factors naturally extend from the approval of a new nanomedicines to the evaluation and approval process for generic and follow-on copies of nanomedicines ${ }^{14,17}$ For instance, despite the originator and generic formulations having similar plasma PK profiles that met criteria for bioequivalence, a reduction in tumor activity was observed in a pre-clinical model with the generic liposomal doxorubicin compared to Doxil. ${ }^{18}$ In a case-control study in recurrent ovarian cancer only $4.3 \%$ of patients responded to the generic "Lipidox" versus $18 \%$ of patients receiving Doxil. ${ }^{19}$ Because the, proprietary process of manufacturing nanomedicines dictates product composition, it is nearly impossible to manufacture an exact copy of the reference listed drug. ${ }^{14,17}$ However, despite these challenges, many product specific guidance documents from the FDA do not propose bioequivalence evaluation methods that cover all of the challenges outlined in the nanomedicine guidance. ${ }^{5}$ Therefore, it is critical for student pharmacists to learn about the gaps in regulatory science for current nanomedicines in their curriculum and bring these skills into their practice. Pharmacists also need to be aware of new scientific discoveries that affect the regulatory evaluation of nanomedicines. They will be the primary member of the interdisciplinary team that has adequate training in pharmaceutical science to synthesize data on known research gaps and new discoveries to apply these concepts to clinical therapeutic decision making and formulary management.

\section{Nanomedicine in Pharmacy Education}

It has become apparent, within the past few years; that nanomedicine has become one of the driving forces of therapeutic and diagnostic developments within the medical field. Furthermore, the regulatory approval of nanomedicine drug products is projected to exponentially grow over the next two decades. ${ }^{6}$ There is also an upward trajectory to clinically utilize nanodiagnostics and nanotherapeutics to profoundly enhance the bench-to bedside approach in patient care. ${ }^{7,20}$ 
Therefore, it has become imperative for both current and future pharmacists to be adequately familiarized and equipped with essential knowledge of nanotechnology, particularly those related with medical applications, to face the developing demands and challenges in their professional practice. Fundamentally, part of the current educational reform within professional pharmacy programs is to integrate foundational science and clinical science education. Thus, these efforts need to effectively incorporate education and training in emerging technological and scientific medical advances, namely nanomedicines and nanodiagnostics, because of their increasing impact on health care. This will not only prepare Pharm. D. professionals better for clinical practice, but also expands their career opportunities. ${ }^{20}$ The 2016 Standards from ACPE, as well as their underlying 2013 CAPE Supplemental Educational Outcomes, underpin foundational science knowledge as an essential framework for understanding clinical knowledge. Hence, in view of CAPE Domain 1 learning objectives, the constructive integration of nanomedicine topics and concepts into foundational pharmaceutical sciences is needed, in order for the pharmacy "learner" to evaluate future advances in medicine, and explain how specific drugs or drug classes work and evaluate their potential value in individuals and populations. Additional pharmacy education data also indicated pharmacy learners seem to approach the safety of nanomedicine with caution, despite positive attitudes and perceptions towards the utilization of nanotechnologies, and suggested teaching nanomedicine focused course material in the curricula. ${ }^{20-23}$

Nanotechnology knowledge integration can be explored at both curricular and co-curricular levels, to provide opportunities for students to gradually construct integrated basic and clinical science knowledge. Key concepts of nanosize surface effects, and quantum mechanical effects should be introduced early on within the pharmaceutics topics, emphasizing the behavior of material at the nanoscale and how it correlates to the physicochemical properties of final nano-pharmaceutical formulation. ${ }^{7}$ The application of these nanotechnology concepts in pharmaceutical dosage courses is also facilitated through many dedicated textbook chapters, in their recently published editions. Within the biopharmaceutics and pharmacokinetics curriculum, nano-scale drug properties can be compared with conventional ones, in terms of routes of drug administration, localized and systemic tissue absorption/permeability, biodistribution, and pharmacokinetics profiles. With nanomedicines constituting almost 10\% of oncology drugs today, the enhanced tumor delivery of nanomedicines can be presented in relevant pharmacology and pharmacotherapy content via commercial nanomedicines, such as Doxil, for passive targeting of Kaposi Sarcoma, and Abraxane, as safer alternative to conventional paclitaxel in ovarian and breast carcinoma protocols. Clinical knowledge framework activities should also conceptualize foundational nanomedicine content and illustrate clinical relevance, via learning objectives and clinical case examples. Elective courses and joint (industrial-clinical) Advanced Pharmacy Practice Experiences for interested students, focused on the design and clinical applications of nanotechnology-based medical products, can integrate, and apply knowledge from both pharmaceutical and clinical foundational sciences to evaluate the scientific literature, explain drug action, solve therapeutic problems, and advance population health and patient-centered care. ${ }^{20-23} \mathrm{~A}$ variety of co-curricular activities can be also employed to further integrate basic science nanomedicine concepts with clinical decision-making. These may include early non-patient care related interprofessional education/collaboration/ innovation (eg, research activities, independent study), medical technology focused seminar series, or meetings. Additionally, professional service organizations related to nano-pharmaceutical career training and post-graduate education, such as Industry Pharmacist Organization (IPhO) student chapters, and Pharmaceutical Industry Fellowship Programs offer venues where interested trainee pharmacists could work in team-based learning formats to work through clinical cases that integrates the complex pharmacokinetic and pharmacodynamic principles associated with nanopharmaceuticals. ${ }^{21,}{ }^{23}$ Considering the imminent medical importance, basic and applied nanomedicine education needs to be proactively integrated within professional pharmacy degree programs, potentially at various levels within the ACPE-structured curricular and co-curricular activities. $^{21,23}$

It is clear that the nanomedicine domain is expanding at a rapid trajectory. Because of the complexity of this class of drugs, pharmacists represent the discipline with the best aptitude to integrate pharmaceutical science, regulatory evaluation and clinical data to lead decision making for patient care. Given the application of nanomedicines to a wide and diverse array of disease states, Doctor of Pharmacy curricula should be forward thinking in their approach to including and expanding the coverage of nanomedicines. Given the density of material in the Doctor of Pharmacy program, innovative techniques such as co-curricular electives, student organization initiatives and certificate programs can effectively provide didactic education on key nanomedicine concepts.

\section{REFERENCES}

1. Ventola CL. Progress in Nanomedicine: Approved and Investigational Nanodrugs. P T. Dec 2017;42(12):742-55.

2. Taniguchi N. On the Basic Concept of Nanotechnology. Proc Int Conf Prod Engin. Part II. Tokyo 1974:18-23. 
3. Nano 101: Nanotechnology, what it is and how it works. . The United States National Nanotechnology Initiative. www.nano.gov/nanotech-101/what. Accessed July 1, 2020.

4. Nanotechnology - Over a Decade of Progress and Innovation: A Report by the U.S. Food and Drug Administration. U.S. Department of Health and Human Services. July 2020.

www.fda.gov/media/140395/download. Accessed October 23, 2020.

5. Drug Products, Including Biological Products, That Contain Nanomaterials - Draft Guidance for Industry issued by: Center for Drug Evaluation and Research, U.S. Food and Drug Administration, Docket Number: FDA-2017D-0759. In: Federal Register NA, ed. 82. Rockville, MD: Office of the Federal Register; 2017:60019.

6. Weissig V, Guzman-Villanueva D. Nanopharmaceuticals (part 2): products in the pipeline. Int J Nanomedicine. 2015;10:1245-57.

7. Mitchell MJ, Billingsley MM, Haley RM, Wechsler ME, Peppas NA, Langer R. Engineering precision nanoparticles for drug delivery. Nat Rev Drug Discov. Dec 42020.

8. $\quad$ Nanomedicine and the COVID-19 vaccines. Nat Nanotechnol. Dec 2020;15(12):963.

9. Liposomal Doxorubicin Market Size, Share, Industry Report, 2013-2024. Grand View Research Inc. https://www.grandviewresearch.com/industry-analysis/liposomal-doxorubicin-market. Accessed January 4, 2021.

10. El-Kilani Z FK, Mulcahy A, and Bosworth, A. Medicare FFSPart B and International Drug Prices: A Comparison of the Top 50 Drugs. Washington, DC: Office of the Assistant Secretary for Planning and Evaluation, U.S. Department of Health and Human Services; 2020:1-29.

11. Barenholz Y. Doxil(R)--the first FDA-approved nano-drug: lessons learned. J Control Release. Jun 10 2012;160(2):117-34.

12. Lawrie TA, Rabbie R, Thoma C, Morrison J. Pegylated liposomal doxorubicin for first-line treatment of epithelial ovarian cancer. Cochrane Database Syst Rev. Oct 21 2013(10):CD010482.

13. Cornely OA, Alastruey-Izquierdo A, Arenz D, et al. Global guideline for the diagnosis and management of mucormycosis: an initiative of the European Confederation of Medical Mycology in cooperation with the Mycoses Study Group Education and Research Consortium. Lancet Infect Dis. Dec 2019;19(12):e405-e21.

14. Nikravesh N, Borchard G, Hofmann H, Philipp E, Fluhmann B, Wick P. Factors influencing safety and efficacy of intravenous iron-carbohydrate nanomedicines: From production to clinical practice. Nanomedicine. Jun 2020;26:102178.

15. Tyner KM, Zheng N, Choi S, et al. How Has CDER Prepared for the Nano Revolution? A Review of Risk Assessment, Regulatory Research, and Guidance Activities. AAPS J. Jul 2017;19(4):1071-83.

16. Pai AB. Evaluating plasma pharmacokinetics of intravenous iron formulations: judging books by their covers? Clin Pharmacokinet. Apr 2015;54(4):323-4.

17. Muhlebach S. Regulatory challenges of nanomedicines and their follow-on versions: A generic or similar approach? Adv Drug Deliv Rev. Jun 2018;131:122-31.

18. Smith JA, Mathew L, Burney M, Nyshadham P, Coleman RL. Equivalency challenge: Evaluation of Lipodox(R) as the generic equivalent for Doxil(R) in a human ovarian cancer orthotropic mouse model. Gynecol Oncol. May 2016;141(2):357-63.

19. Smith JA, Costales AB, Jaffari M, et al. Is it equivalent? Evaluation of the clinical activity of single agent Lipodox $(\mathrm{R})$ compared to single agent Doxil(R) in ovarian cancer treatment. J Oncol Pharm Pract. Aug 2016;22(4):599-604.

20. Al-Nemrawi NK, AbuAlSamen MM, Alzoubi KH. Awareness about nanotechnology and its applications in drug industry among pharmacy students. Curr Pharm Teach Learn. Mar 2020;12(3):274-80.

21. Piascik P. CAPE outcomes 2013: Building on two decades of advances to guide the future of pharmacy education. Am J Pharm Educ. Oct 14 2013;77(8):160.

22. Greene JM, Fuller KA, Persky AM. Practical Tips for Integrating Clinical Relevance into Foundational Science Courses. Am J Pharm Educ. Jun 2018;82(5):6603.

23. Maerten-Rivera JL, Chen AMH, Augustine J, et al. Co-Curriculum Implementation and Assessment in Accredited Doctor of Pharmacy Programs. Am J Pharm Educ. Mar 2020;84(3):7569. 
Figure 2. Schematic of types of nanomedicines and size comparisons of the various types of nanomedicines. ${ }^{17 *}$

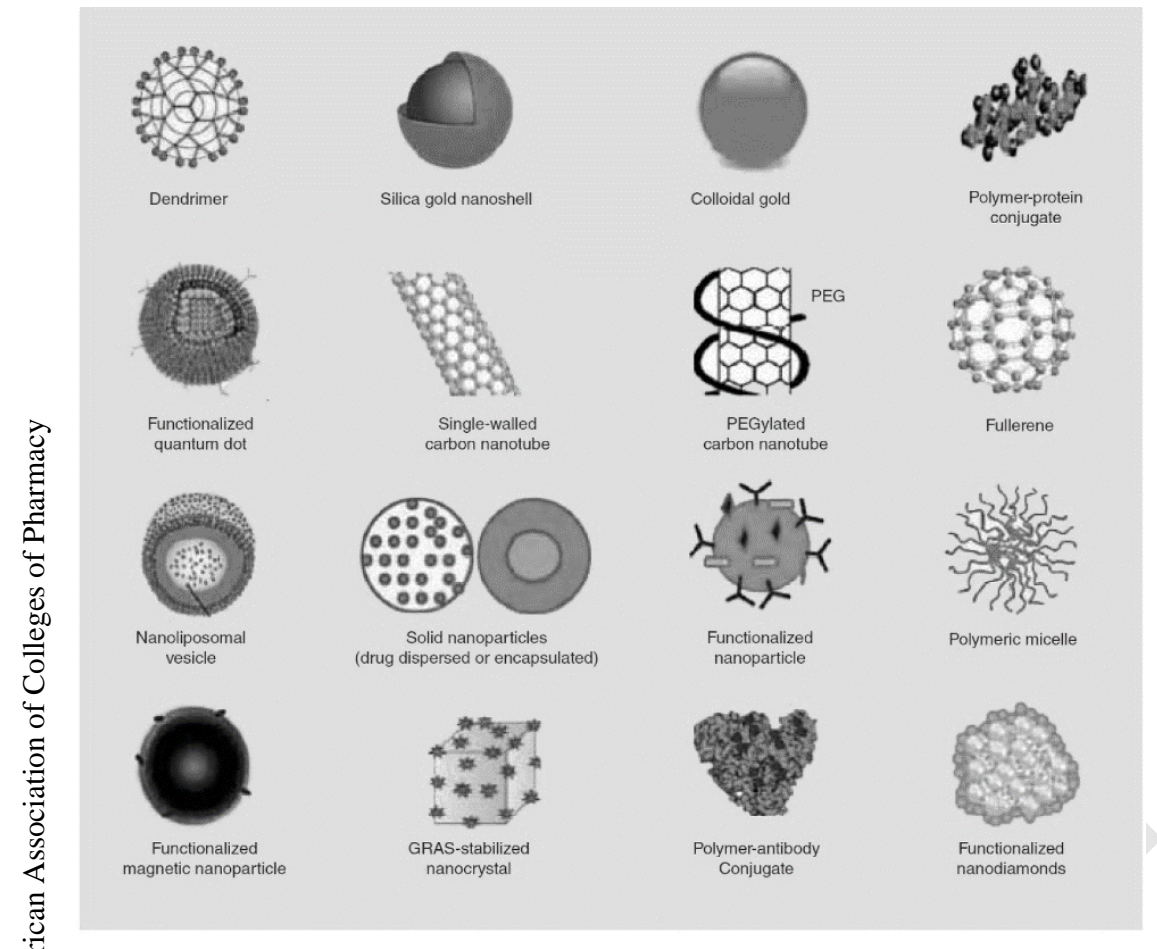

\section{b Size comparison of nanomedicines}

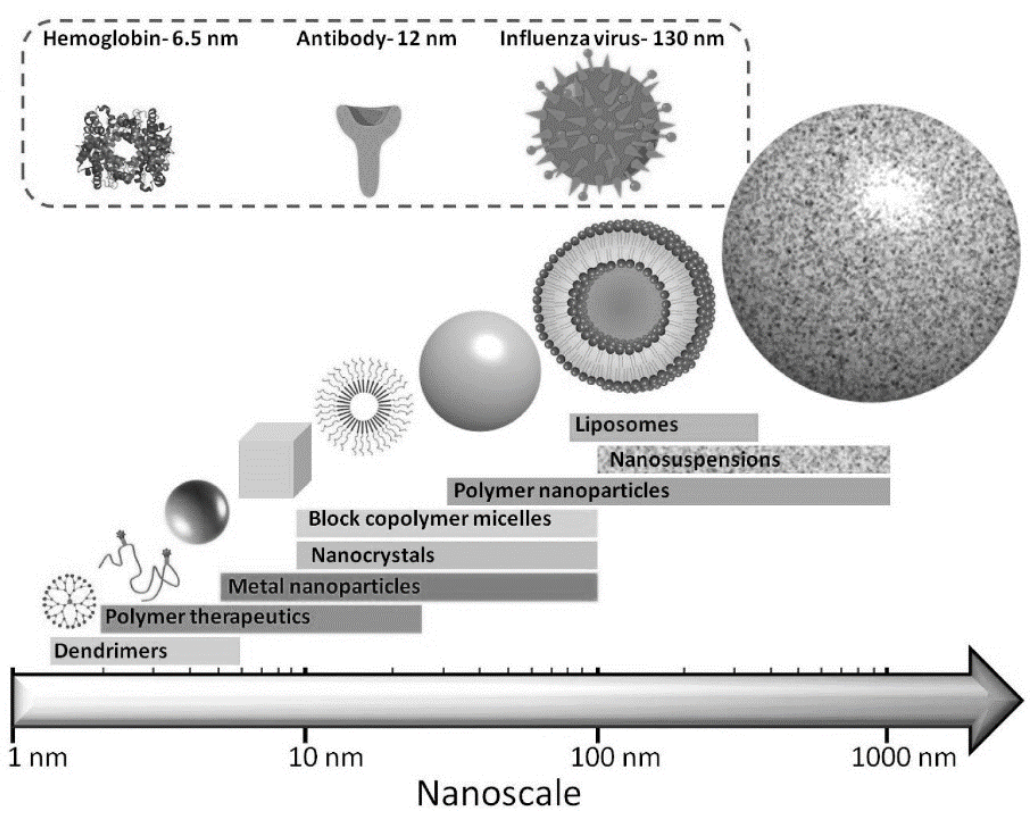

*Figure reproduced under the terms of the Creative Commons CC-BY license 